\section{Vol. 38, Issue 2, April 2011}

\section{Editorial}

85 Looking Back from the Future to the Present: Biopreservation Will Get Us There!

Sputtek, A. (Hamburg); Rowe, A.W. (New York, NY)

\section{Review Articles}

89 Membrane Stability during Biopreservation of Blood Cells

Stoll, C.; Wolkers, W.F. (Hannover)

99 Emerging Role for Use of Liposomes in the Biopreservation of Red Blood Cells

Holovati, J.L.; Acker, J.P. (Edmonton, AB)

107 Cryopreservation of Human Stem Cells for Clinical Application: A Review Hunt, C.J. (South Mimms)

125 Organ Preservation: Current Concepts and New Strategies for the Next Decade

Guibert, E.E. (Rosario); Petrenko, A.Y. (Kharkov); Balaban, C.L. (Rosario); Somov, A.Y. (Kharkov); Rodriguez, J.V. (Rosario); Fuller, B.J. (London)

143 Preservation of Human Cornea Armitage, W.J. (Bristol)

149 Packaging Considerations for Biopreservation Woods, E.J.; Thirumala, S. (Indianapolis, IN)

\section{Commentaries}

157 To the Judgement of the European Court of Justice from December 9, 2010 concerning a National Rule That Bans the Import of Blood Products Which Do Not Originate from Blood Donations Completely Uncompensated von Auer, F. (Königswinter)

\section{Band 38, Heft 2, April 2011}

Editorial

85 Rückblick aus der Zukunft in die Gegenwart: Biologische Kryokonservierung wird uns dorthin bringen!

Sputtek, A. (Hamburg); Rowe, A.W. (New York, NY)

\section{Übersichtsarbeiten}

89 Membranstabilität während der Biokonservierung von Blutzellen

Stoll, C.; Wolkers, W.F. (Hannover)

99 Neue Perspektiven für die Nutzung von Liposomen zur biologischen Konservierung von Erythrozyten

Holovati, J.L.; Acker, J.P. (Edmonton, AB)

107 Kryokonservierung von menschlichen Stammzellen für die klinische Anwendung: Eine Übersicht

Hunt, C.J. (South Mimms)

125 Organkonservierung: Derzeitige Konzepte und neue Strategien für das kommende Jahrzehnt Guibert, E.E. (Rosario); Petrenko, A.Y. (Kharkov); Balaban, C.L. (Rosario); Somov, A.Y. (Kharkov); Rodriguez, J.V. (Rosario); Fuller, B.J. (London)

143 Konservierung menschlicher Augenhornhäute Armitage, W.J. (Bristol)

149 Biolologische Konservierung: Auch eine Frage des Behältnisses

Woods, E.J.; Thirumala, S. (Indianapolis, IN)

Kommentare

157 Zum Urteil des Europäischen Gerichtshofs vom 9. Dezember 2010 zu einer nationalen Regelung, die die Einfuhr von Blutprodukten verbietet, die nicht aus gänzlich unbezahlt erfolgten Blutspenden stammen von Auer, F. (Königswinter)

\section{KARGER}

Fax +497614520714

Information@Karger.de

www.karger.com 


\section{Vol. 38, Issue 2, April 2011}

159 'Legally Binding Force' of Guidelines Regarding the Collection of Blood and Blood Components and the Application of Blood Products

(Hemotherapy) after Amendment of the German Transfusion Law

Hasskarl, H.; Bakhschai, B. (Ludwigshafen)

165 News / Ticker

166 Meetings and Conferences

168 Guidelines for Authors

87 Imprint

\section{Band 38, Heft 2, April 2011}

159 «Rechtliche Verbindlichkeit» der Richtlinien zur Gewinnung von Blut und Blutbestandteilen und zur Anwendung von Blutprodukten

(Hämotherapie) nach der Novellierung des Transfusionsgesetzes

Hasskarl, H.; Bakhschai, B. (Ludwigshafen)

165 News / Ticker

166 Tagungen und Kongresse

168 Hinweise für Autoren

87 Impressum 